\title{
Metabolic Syndrome and Psoriasis: A Case- Control Study in Kabul, Afghanistan
}

This article was published in the following Dove Press journal:

Diabetes, Metabolic Syndrome and Obesity: Targets and Therapy

\author{
Ahmad Khalid Aalemi $\mathbb{D}^{1,2}$ \\ Mohammad Barin Bahain ${ }^{3}$ \\ Abdul Ghafar Hamdard ${ }^{2}$ \\ 'Department of Epidemiology, Kabul \\ University of Medical Sciences, Kabul, \\ Afghanistan; ${ }^{2}$ Department of \\ Dermatology, Maiwand Teaching \\ Hospital, Kabul University of Medical \\ Sciences, Kabul, Afghanistan; \\ ${ }^{3}$ Department of Pharmacology, Kabul \\ University of Medical Sciences, Kabul, \\ Afghanistan
}

Correspondence: Ahmad Khalid Aalemi Department of Epidemiology, Kabul University of Medical Sciences, Kabul, I00I, Afghanistan

Tel +9374794758I

Email aalemi_100@yahoo.com
Background: Psoriasis is a chronic inflammatory disease that is not limited to the skin. Recently, numerous studies have shown a positive association between metabolic syndrome and psoriasis.

Objective: The current study aimed to examine the association of metabolic syndrome with psoriasis in an Afghan population.

Methods: This was a case-control study including 114 patients with psoriasis and 114 controls aged $\geq 18$ years admitted to the dermatology department of Maiwand Teaching Hospital in Kabul, Afghanistan. Height, weight, blood pressure, and waist circumference were measured in all subjects. Blood glucose, triglyceride, cholesterol, and high-density lipoprotein cholesterol levels were tested following overnight fasting. The modified National Cholesterol Education Program Adult Treatment Panel III criteria were used for the diagnosis of metabolic syndrome.

Results: In total, $51.8 \%$ of the cases and $44.7 \%$ of the controls were male. The average age of participants was $33.4 \pm 13.1$ years in the case group and $41.1 \pm 15.4$ years in the control group. The average duration of disease for psoriasis was 4.2 years with 5.6 years SD. The average PASI was 10.8 with 5.1 SD. More than half of the cases $(62.3 \%)$ had moderate to severe psoriasis and $37.7 \%$ had mild psoriasis. The prevalence of metabolic syndrome was higher among patients with psoriasis compared to controls $(36.8 \%$ vs $21.1 \%)$ with OR of 2.18 ( $p=0.009)$. In addition, overweight/obesity was more prevalent among cases compared to controls $(65.8 \%$ vs $41.2 \%)$ with OR of $2.74(p<0.001)$, whereas the waist circumference was not significantly different between the two groups. Furthermore, the mean levels of total cholesterol, triglyceride, and fasting blood glucose were also higher among patients with psoriasis compared to controls.

Conclusion: The results of the study confirm the association between psoriasis and metabolic syndrome. Hence, screening psoriatic patients for metabolic syndrome should be considered.

Keywords: psoriasis, metabolic syndrome, hypertension, dyslipidemia, obesity, case-control study, Kabul

\section{Introduction}

Psoriasis is a chronic recurrent inflammatory skin disease. ${ }^{1}$ It affects $2 \%$ to $4 \%$ of the general population. ${ }^{2}$ The chronic inflammatory natures of psoriasis predispose the patient to several comorbidities, such as obesity, diabetes, and metabolic syndrome. ${ }^{3}$ Psoriasis comorbidities have a significant impact on the management and quality of life of patients with psoriasis. ${ }^{4,5}$ Numerous studies have shown an increased frequency of metabolic syndrome and its components among patients with psoriasis. ${ }^{6-11}$ A meta-analysis of 35 observational studies found that patients with psoriasis were 2.14 (95\% CI 1.84-2.48) times more likely to have metabolic syndrome than the general population. ${ }^{2}$ A study in South Africa found that patients with psoriasis were more likely to have metabolic 
syndrome than patients without psoriasis $(52.4 \%$ vs $33.7 \%$, $p=0.007) .{ }^{12}$ Salunke et al found that metabolic syndrome was more common among Indian patients with psoriasis compared to controls $(38.9 \%$ vs $21.0 \%, p=0.007) .{ }^{8}$ Farshchian et al found that the mean of triglyceride, total cholesterol, blood pressure, and body mass index (BMI) were higher among Iranian patients with psoriasis compared to controls. ${ }^{13}$ Gui et al found a higher prevalence of metabolic syndrome among patients with psoriasis compared to controls in a Chinese population $(14.3 \%$ vs $10 . \%, p=0.001)$ with $\mathrm{OR}$ of 1.5 . $^{1}$ However, the mechanism of this association is not fully understood. Pro-inflammatory cytokines and immunological mediators are thought to play a role in this mechanism. ${ }^{14}$ It is suggested that patients with psoriasis should be closely monitored for metabolic syndrome. ${ }^{15}$ Significant variations are seen in the characteristics of both psoriasis and metabolic syndrome among different geographic areas and ethnic groups. No such study has been conducted in Afghanistan. The present study aimed to investigate the association between metabolic syndrome and psoriasis in Kabul citizens, which is the first study of its kind.

\section{Methodology}

This is a case-control study conducted at the dermatology department of Maiwand Teaching Hospital in Kabul City, from December 2018 to July 2019. This study was conducted in accordance with the Declaration of Helsinki. Ethical clearance was provided by the Institutional Review Board of the Kabul University of Medical Sciences. Written informed consent was obtained from the subjects prior to enrollment. The sample size, calculated by Epi Info, was 228 subjects considering $95 \%$ confidence interval, power $=80 \%$, odds ratio $(\mathrm{OR})=2.39$, and the percent of control to exposed was $21 \% .^{8}$ In total, 114 patients with clinical diagnosis of plaque psoriasis aged $\geq 18$ years were included in the case group. The control group consisted of 114 subjects who visited the dermatology department for conditions other than psoriasis and who did not have psoriasis. Subjects receiving systemic treatment such as steroids, immunosuppressants, and retinoids for at least six weeks before enrolment were excluded from the study. Demographic data (such as sex, age, education, occupation, smoking, alcohol consumption, drug abuse, etc.) and information on family history of diabetes, hypertension, and skin diseases were collected using a preadministered questionnaire. Clinical aspect of psoriasis (disease duration and joint involvement), the Psoriasis
Area and Severity Index (PASI), blood pressure, waist circumference, and body mass index (BMI) were obtained through physical examination.

Weight and height were measured using a standardized procedure with light clothing and without shoes. BMI was calculated as weight $(\mathrm{kg})$ divided by height squared $\left(\mathrm{m}^{2}\right)$. The blood pressure was measured in a sitting position from the right arm of subjects. Two readings were taken at a 30 minute interval and the average was used. The waist circumference was measured with a flexible tape positioned at the level between the iliac crest and lower costal border. Blood samples were taken 12 hours after overnight fasting for the measurement of blood sugar and lipid levels. The latter (serum cholesterol, triglyceride, and high-density lipoprotein (HDL) cholesterol) was measured by enzymatic/colorimetric method.

The severity of psoriasis was identified using the PASI score. A score less than 10 was considered as mild psoriasis and above 10 was classified as moderate to severe psoriasis. ${ }^{16}$ The criteria for diagnosis of metabolic syndrome were those recommended by the National Cholesterol Education Program - Adult Treatment Panel III which is defined by the presence of at least three of the following components: ${ }^{17}$

1. Waist circumference $\geq 90 \mathrm{~cm}$ in Asian males and $\geq 80 \mathrm{~cm}$ in Asian females;

2. Serum triglycerides $\geq 150 \mathrm{mg} / \mathrm{dl}$ (or on treatment for raised triglycerides);

3. HDL cholesterol $<40 \mathrm{mg} / \mathrm{dL}$ in males and $<50 \mathrm{mg} /$ $\mathrm{dL}$ in females (or on treatment for reduced HDL-c);

4. Blood pressure: systolic $\geq 130$ and/or diastolic $\geq 85$ $\mathrm{mmHg}$ (or on treatment for hypertension);

5. Fasting glucose $\geq 100 \mathrm{mg} / \mathrm{dL}$ (or on treatment for increased blood glucose).

\section{Statistical Analysis}

Statistical analysis was performed using Statistical Package for Social Science (SPSS) version 22.0. The socio-demographic characteristics were presented as percentage, mean, and standard deviation. Categorical variables were compared between the cases and controls using the chi-square test or Fisher's exact test, and continuous variables were compared by independent $t$-test. Odds Ratio (OR) and $95 \%$ CI were calculated. A $p$-value $<0.05$ was considered statistically significant. 


\section{Results}

Table 1 shows the socio-demographic characteristics of the subjects according to their case and control status. The mean age of patients with psoriasis was 33.4 years with 13.1 years standard deviation (SD) and 41.1 years with 15.4 years SD for controls $(p<0.005)$. Almost half of the patients with psoriasis (51.8\%) and controls (44.7\%) were male $(p=0.28)$. More than half of the patients with psoriasis $(54.4 \%)$ and controls $(55.3 \%)$ were illiterate $(p=0.89)$. Most of the patients with psoriasis $(71.1 \%)$ and controls $(80.7 \%)$

Table I Socio-Demographic Characteristics of the Participants According to Their Case-Control Status

\begin{tabular}{|c|c|c|c|c|c|c|c|}
\hline & \multicolumn{2}{|c|}{ Case } & \multicolumn{2}{|c|}{ Control } & \multicolumn{2}{|c|}{ Overall } & \multirow[t]{2}{*}{$p$ value } \\
\hline & $\mathbf{n}$ & $\%$ & $\mathbf{n}$ & $\%$ & $\mathbf{n}$ & $\%$ & \\
\hline Age, years & \multicolumn{2}{|c|}{$33.4 \pm 13.1$} & \multicolumn{2}{|c|}{$4 I .1 \pm 15.4$} & \multicolumn{2}{|c|}{$37.2 \pm 14.7$} & $<0.005$ \\
\hline$<20$ & 6 & 5.3 & 5 & 4.4 & 11 & 4.8 & 0.016 \\
\hline $20-29$ & 49 & 43.0 & 30 & 26.3 & 79 & 34.6 & \\
\hline $30-39$ & 20 & 17.5 & 14 & 12.3 & 34 & 14.9 & \\
\hline $40-49$ & 22 & 19.3 & 28 & 24.6 & 50 & 21.9 & \\
\hline $50-59$ & 9 & 7.9 & 18 & 15.8 & 27 & 11.8 & \\
\hline$\geq 60$ & 8 & 7.0 & 19 & 16.7 & 27 & 11.8 & \\
\hline Sex & & & & & & & 0.28 \\
\hline Male & 59 & 51.8 & 51 & 44.7 & 110 & 48.2 & \\
\hline Female & 55 & 48.2 & 63 & 55.3 & 118 & 51.8 & \\
\hline Education status & & & & & & & 0.89 \\
\hline Illiterate & 62 & 54.4 & 63 & 55.3 & 125 & 54.8 & \\
\hline Literate & 52 & 45.6 & 51 & 44.7 & 103 & 45.2 & \\
\hline Marital status & & & & & & & 0.08 \\
\hline Single & 33 & 28.9 & 22 & 19.3 & 55 & 24.1 & \\
\hline Married & 81 & 71.1 & 92 & 80.7 & 173 & 75.9 & \\
\hline Physical exercise & & & & & & & 0.002 \\
\hline No & 95 & 83.3 & 75 & 65.8 & 170 & 74.6 & \\
\hline Occasionally/regularly & 19 & 16.7 & 39 & 34.2 & 58 & 25.4 & \\
\hline Life satisfaction & & & & & & & $<0.001$ \\
\hline Dissatisfied & 33 & 28.9 & 7 & 6.1 & 40 & 17.5 & \\
\hline $\begin{array}{l}\text { Neither satisfied nor } \\
\text { dissatisfied }\end{array}$ & 50 & 43.9 & 2 & 1.8 & 52 & 22.8 & \\
\hline Satisfied & 31 & 27.2 & 105 & 92.1 & 136 & 59.6 & \\
\hline Smoking & & & & & & & 0.19 \\
\hline Never & 111 & 97.4 & 107 & 93.9 & 218 & 95.6 & \\
\hline Ex/current smoker & 3 & 2.6 & 7 & 6.1 & 10 & 4.4 & \\
\hline Smoker in a family & & & & & & & 0.23 \\
\hline No & 92 & 80.7 & 101 & 88.6 & 193 & 86.6 & \\
\hline Spouse & 10 & 8.8 & 5 & 4.4 & 15 & 6.6 & \\
\hline Father/sibling & 12 & 10.5 & 8 & 7.0 & 20 & 8.8 & \\
\hline Family history & & & & & & & \\
\hline Hypertension & 28 & 24.6 & 11 & 9.6 & 39 & 17.1 & 0.003 \\
\hline Diabetes & 10 & 8.8 & 5 & 4.4 & 15 & 6.6 & 0.18 \\
\hline Skin disorders & 12 & 10.5 & 10 & 8.8 & 22 & 9.6 & 0.65 \\
\hline
\end{tabular}

were married $(p=0.08)$. Occasional or regular physical exercise was more prevalent among controls (34.2\%) compared to cases $(16.7 \%)(p=0.002)$. The patients with psoriasis were more unsatisfied with life (28.9\%) compared to controls $(6.1 \%)(p<0.001)$. The prevalence of smoking was very low in both groups and the difference was not statistically significant. One-fifth of the cases (19.3\%) had a smoker in their family and this was higher compared to controls $(11.4 \%)$ but the difference was not statistically significant. Family history of hypertension was higher among cases (24.6\%) compared to controls $(9.6 \%) \quad(p=0.003)$. Family history of diabetes and skin disorders was not different among the two groups.

The average duration of disease was 4.2 years with 5.6 years SD. The average PASI was 10.8 with 5.1 SD. More than half of the patients with psoriasis $(62.3 \%)$ had moderate to severe psoriasis while $37.7 \%$ of them had mild psoriasis.

Table 2 shows the prevalence of metabolic syndrome and its components according to the case and control status. The prevalence of metabolic syndrome was higher among patients with psoriasis $(36.8 \%)$ compared to controls $(21.1 \%)$ with OR of $2.18(p=0.009)$. Furthermore, overweight/obesity was more common among cases (65.8\%) compared to controls (41.2\%) with OR of 2.74 $(p<0.001)$. The waist circumference was higher among cases $(41.2 \%)$ relative to controls $(31.6 \%)$ but the difference was not statistically significant. We also observed a higher prevalence of high blood pressure among the psoriasis group (53.5\%) compared to controls (33.3\%) with OR of $2.30(p=0.002)$. In addition to that, the psoriasis group had higher prevalence of diabetes $(32.5 \%)$ compared to controls (15.8\%) with OR of 2.56 $(p=0.003)$. High level of triglyceride was more prevalent among cases $(66.7 \%)$ compared to controls $(35.1 \%)$ with OR of $3.70(p<0.001)$. The low level of HDL cholesterol was more common among the psoriasis group (32.5\%) compared to controls $(19.3 \%)$ with OR of $2.0(p=0.023)$. The mean levels of total cholesterol, triglyceride, and fasting blood glucose were also higher among patients with psoriasis compared to controls.

Figure 1 shows the prevalence of metabolic syndrome and its components according to the severity of psoriasis. The patients with moderate to severe psoriasis had higher prevalence of metabolic syndrome relative to patients with mild psoriasis $(49.3 \%$ vs $16.3 \%, p<0.001)$. In addition to that, abdominal obesity was more common among moderate to severe psoriasis compared to mild psoriasis $(53.5 \%$ 
Table 2 Distribution of Metabolic Syndrome and Its Components According to the Case and Control Status

\begin{tabular}{|c|c|c|c|c|c|c|c|}
\hline & \multicolumn{2}{|c|}{ Case } & \multicolumn{2}{|c|}{ Control } & \multicolumn{2}{|c|}{ Overall } & \multirow[t]{2}{*}{$p$ value } \\
\hline & $\mathbf{n}$ & $\%$ & $\mathbf{n}$ & $\%$ & $\mathbf{n}$ & $\%$ & \\
\hline Metabolic syndrome & \multicolumn{6}{|c|}{$\mathrm{OR}=2.18,95 \% \mathrm{Cl}=1.2 \mathrm{I}-3.94$} & 0.009 \\
\hline No & 72 & 63.2 & 90 & 78.9 & 162 & 71.1 & \\
\hline Yes & 42 & 36.8 & 24 & 21.1 & 66 & 28.9 & \\
\hline Obesity status & \multicolumn{6}{|c|}{$\mathrm{OR}=2.74,95 \% \mathrm{Cl}=1.60-4.69$} & $<0.001$ \\
\hline Underweight/normal & 39 & 34.2 & 67 & 58.8 & 106 & 46.5 & \\
\hline Overweight/obese & 75 & 65.8 & 47 & 41.2 & 122 & 53.5 & \\
\hline Waist circumference & \multicolumn{6}{|c|}{$\mathrm{OR}=1.52,95 \% \mathrm{Cl}=0.88-2.6 \mathrm{I}$} & 0.13 \\
\hline$<90 \mathrm{~cm}($ male) $/<80 \mathrm{~cm}$ (female) & 67 & 58.8 & 78 & 68.4 & 145 & 63.6 & \\
\hline$\geq 90 \mathrm{~cm}($ male $) / \geq 80 \mathrm{~cm}$ (female) & 47 & 41.2 & 36 & 31.6 & 83 & 36.4 & \\
\hline Blood pressure, systolic or diastolic & \multicolumn{6}{|c|}{$\mathrm{OR}=2.30,95 \% \mathrm{Cl}=1.34-3.93$} & 0.002 \\
\hline$<130 \mathrm{mmHg}$ or $<85 \mathrm{mmHg}$ & 53 & 46.5 & 76 & 66.7 & 129 & 56.6 & \\
\hline$\geq 130 \mathrm{mmHg}$ or $\geq 85 \mathrm{mmHg}$ & 61 & 53.5 & 38 & 33.3 & 99 & 43.4 & \\
\hline Blood glucose & \multicolumn{6}{|c|}{$\mathrm{OR}=2.56,95 \% \mathrm{Cl}=1.35-4.85$} & 0.003 \\
\hline$<100 \mathrm{mg} / \mathrm{dL}$ & 77 & 67.5 & 96 & 84.2 & 173 & 75.9 & \\
\hline$\geq 100 \mathrm{mg} / \mathrm{dL}$ & 37 & 32.5 & 18 & 15.8 & 55 & 24.1 & \\
\hline Triglyceride & \multicolumn{6}{|c|}{$\mathrm{OR}=3.70,95 \% \mathrm{Cl}=2.14-6.39$} & $<0.001$ \\
\hline$<150 \mathrm{mg} / \mathrm{dL}$ & 38 & 33.3 & 74 & 64.9 & 112 & 49.1 & \\
\hline$\geq 150 \mathrm{mg} / \mathrm{dL}$ & 76 & 66.7 & 40 & 35.1 & 116 & 50.9 & \\
\hline HDL cholesterol & \multicolumn{6}{|c|}{$\mathrm{OR}=2.00,95 \% \mathrm{Cl}=1.09-3.69$} & 0.023 \\
\hline$\geq 40 \mathrm{mg} / \mathrm{dL}$ (male) $/ \geq 50 \mathrm{mg} / \mathrm{dL}$ (female) & 77 & 67.5 & 92 & 80.7 & 169 & 74.1 & \\
\hline$<40 \mathrm{mg} / \mathrm{dL}$ (male) $/<50 \mathrm{mg} / \mathrm{dL}$ (female) & 37 & 32.5 & 22 & 19.3 & 59 & 25.9 & \\
\hline Blood glucose, mean $\pm \mathrm{SD}, \mathrm{mg} / \mathrm{dL}$ & \multicolumn{2}{|c|}{$100.5 \pm 31.2$} & \multicolumn{2}{|c|}{$91.9 \pm 27.9$} & \multicolumn{2}{|c|}{$96.2 \pm 29.8$} & 0.03 \\
\hline Triglyceride, mean $\pm \mathrm{SD}, \mathrm{mg} / \mathrm{dL}$ & \multicolumn{2}{|c|}{$178.3 \pm 62.8$} & \multicolumn{2}{|c|}{$157.7 \pm 44.8$} & \multicolumn{2}{|c|}{$168.0 \pm 55.4$} & 0.005 \\
\hline Total cholesterol, mean $\pm \mathrm{SD}, \mathrm{mg} / \mathrm{dL}$ & \multicolumn{2}{|c|}{$171.0 \pm 26.4$} & \multicolumn{2}{|c|}{$159.7 \pm 30.3$} & \multicolumn{2}{|c|}{$165.4 \pm 28.9$} & 0.003 \\
\hline
\end{tabular}

vs $20.9 \%, p<0.005)$. Furthermore, dyslipidemia was also more prevalent among patients with moderate to severe psoriasis than mild psoriasis $(77.6 \%$ vs $48.8 \%, p<0.005$; hypertriglyceridemia and $46.5 \%$ vs $9.3 \%, p<0.001$; low HDL cholesterol). However, there was no difference in the prevalence of hypertension and diabetes by severity of psoriasis.

\section{Discussion}

The association between psoriasis and metabolic syndrome has been investigated with considerable variability in the results in a large number of studies with different study designs in different parts of the world. The results of our study show a higher prevalence of metabolic syndrome and its components among Afghan patients with psoriasis relative to controls.
A recent study conducted in Spain showed that metabolic syndrome was more prevalent among patients with psoriasis compared to controls $(28.3 \%$ vs $15.1 \%$, $p<0.001$; OR 2.21). ${ }^{18}$ Goolam Mahyoodeen et $\mathrm{al}^{12}$ found that the prevalence of metabolic syndrome was higher among patients with psoriasis compared to controls in a South African population $(52.4 \%$ vs $33.7 \%$; $p=0.007$ ). According to the population-based study in Norway conducted by Snekvik et al, metabolic syndrome was more common in the psoriasis group than controls (31.1\% vs $23.0 \%, p<0.001) .{ }^{6}$ Gui et al concluded that the prevalence of psoriasis was higher among Chinese patients with psoriasis compared to controls $(14.3 \%$ vs $10.0 \%, p=0.001) .{ }^{1}$ Ferdinando et $\mathrm{al}^{3}$ also found a higher prevalence of metabolic syndrome in the psoriasis group 


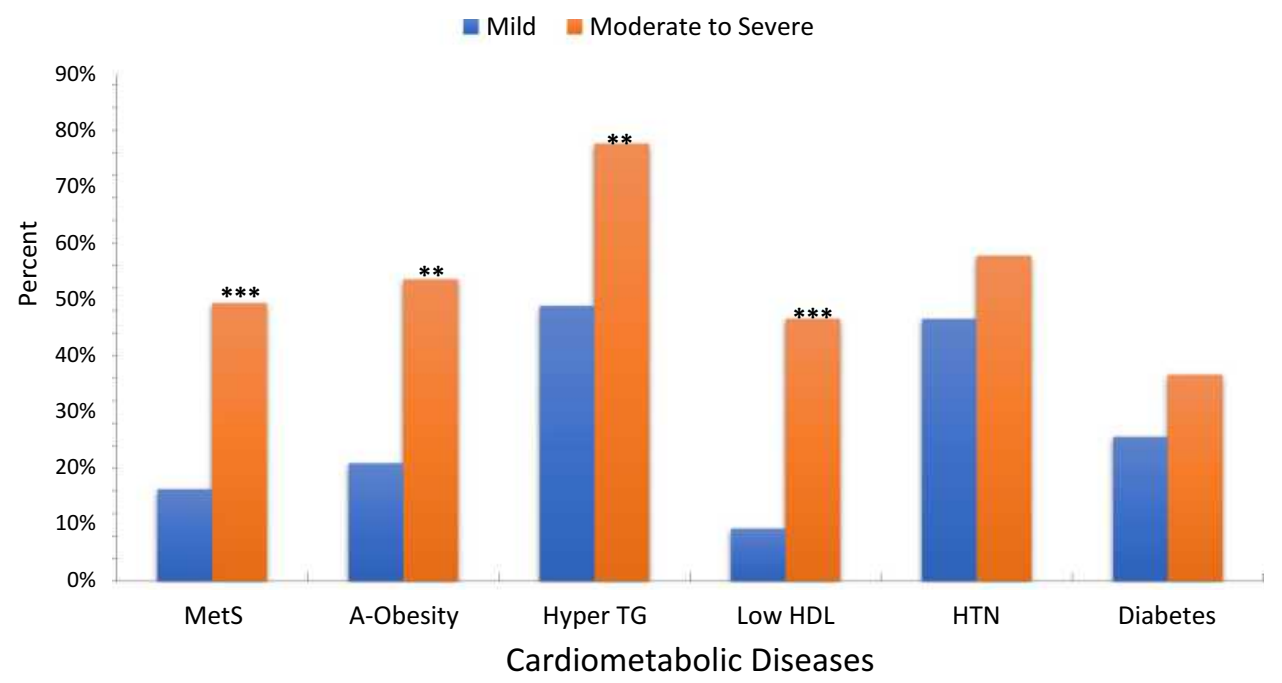

Figure I Prevalence of metabolic syndrome and its components by severity of psoriasis in Afghan patients. $* * p<0.005$, $* * * p<0.001$.

Abbreviations: MetS, metabolic syndrome; A-Obesity, abdominal obesity; Hyper TG, hypertriglyceridemia; Low HDL, low high-density lipoprotein; HTN, hypertension.

relative to controls in Brazil (49.4\% vs $35.0 \%, p=0.04$; OR 1.8). However, some studies found no association between psoriasis and metabolic syndrome such as Kim et al $^{19}$ and Orgaz-Molina et al. ${ }^{20}$ These discrepancies might be due to genetic and racial variations, and low prevalence of metabolic syndrome in both groups.

In the current study, we found higher prevalence of metabolic syndrome among patients with psoriasis compared to controls $(36.8 \%$ vs $21.1 \%, p=0.009$; OR 2.2$)$, which is similar to most of the previous studies. The exact mechanism of this association is not fully understood yet but both diseases share multiple inflammatory and cytokine-mediated mechanisms. New evidence suggests that local overproduction of pro-inflammatory mediators in psoriasis may migrate to systemic circulation that may result in systemic insulin resistance, circulatory endothelial dysfunctions, increased oxidative stress, increased angiogenesis, and hypercoagulation, all of which may result in cardiovascular damage.

The study showed that overweight/obesity was more prevalent among patients with psoriasis relative to controls (65.8\% vs $41.2 \%, p<0.001$; OR 2.7), which is in agreement with studies conducted by Singh et al, ${ }^{9}$ Koku Aksu et al, ${ }^{21}$ Itani et al, ${ }^{22}$ and Kim et al. ${ }^{23}$ Whereas, we found no statistical association between waist circumference and psoriasis which is consistent with studies conducted by Akcali et $\mathrm{al}^{24}$ Albareda et al ${ }^{16}$ and Orgaz-Molina et al. ${ }^{20}$ However, this finding is in contrast with studies conducted by Espinoza et al, ${ }^{25}$ Kokpol et al, ${ }^{26}$ and Meziane et al. ${ }^{27}$ The different results might be due to genetic, racial and behavioral differences.
In addition, the study indicated that dyslipidemia was more common among patients with psoriasis compared to controls, which was consistent with findings of studies conducted by Fernandez-Armenteros et $\mathrm{al}^{18}$ Goolam Mahyoodeen et al, ${ }^{12}$ and Gui et al. ${ }^{1}$ Furthermore, we found higher prevalence of hypertension among patients with psoriasis relative to controls, which was in agreement with studies conducted in Iran, ${ }^{13}$ India, ${ }^{9}$ South Africa, ${ }^{12}$ and Poland, ${ }^{10}$ while it was inconsistent with studies conducted in China ${ }^{1}$ and Korea. ${ }^{19,28}$ These discrepancies might be due to racial and cultural differences. We also observed that hyperglycemia was more prevalent among patients with psoriasis than controls. This finding was confirmed by studies conducted by Ghiasi et al in Iran, ${ }^{29}$ Langan et al in the United Kingdom, ${ }^{30}$ and Kokpol et al in Thailand. ${ }^{26}$

Moreover, the study results indicate that metabolic syndrome and dyslipidemia were more common among patients with moderate to severe psoriasis compared to mild psoriasis (Figure 1), which is consistent with studies conducted by Goolam Mahyoodeen et $\mathrm{al}^{12}$ and Milcic et al. ${ }^{31}$ However, some studies found no statistically significant difference in the prevalence of metabolic syndrome by severity of psoriasis. ${ }^{18,32}$ The reason behind this difference might be the small sample size. Moreover, most of the studies that assessed the association of metabolic syndrome with psoriasis did not evaluate the association of metabolic syndrome with severity of psoriasis. ${ }^{1,3,9,33}$

Strengths of this study include: administration of the questionnaire by a dermatologist, using the PASI to define the severity of psoriasis, exclusion of patients with systemic drugs, and to the best of our knowledge this was the 
first study on adult psoriasis conducted in Afghanistan. Limitations of this study include: relatively small sample size, and using a hospital-based case-control design that might not be generalized to patients with psoriasis in the community. In addition, smoking habits might be underreported due to cultural issues.

In conclusion, Afghan patients with psoriasis showed a higher prevalence of metabolic syndrome and its components compared to controls. Therefore, screening for metabolic syndrome is strongly recommended for Afghan psoriatic patients to benefit from early diagnosis and intervention, such as lifestyle changes, weight loss, and treatment of hypertension, diabetes, and dyslipidemia, to reduce the risk of major cardiovascular events.

\section{Disclosure}

The authors report no conflicts of interest in this work.

\section{References}

1. Gui XY, Yu XL, Jin HZ, Zuo YG, Wu C. Prevalence of metabolic syndrome in Chinese psoriasis patients: a hospital-based cross-sectional study. $J$ Diabetes Investig. 2018;9(1):39-43. doi:10.1111/jdi.12663

2. Singh S, Young P, Armstrong AW. An update on psoriasis and metabolic syndrome: a meta-analysis of observational studies. PLoS One. 2017;12(7):e0181039. doi:10.1371/journal.pone.0181039

3. Ferdinando LB, Fukumoto PK, Sanches S, et al. Metabolic syndrome and psoriasis: a study in 97 patients. Rev Assoc Med Bras. 2018;64 (4):368-373. doi:10.1590/1806-9282.64.04.368

4. Furue M, Tsuji G, Chiba T, Kadono T. Cardiovascular and metabolic diseases comorbid with psoriasis: beyond the skin. Intern Med. 2017;56(13):1613-1619. doi:10.2169/internalmedicine.56.8209

5. Greb JE, Goldminz AM, Elder JT, et al. Psoriasis. Nat Rev Dis Primers. 2016;2:16082. doi:10.1038/nrdp.2016.82

6. Snekvik I, Nilsen TIL, Romundstad PR, et al. Psoriasis and cardiovascular disease risk factors: the HUNT Study, Norway. J Eur Acad Dermatol Venereol. 2018;32(5):776-782. doi:10.1111/jdv.14835

7. Adisen E, Uzun S, Erduran F, Gurer MA. Prevalence of smoking, alcohol consumption and metabolic syndrome in patients with psoriasis. An Bras Dermatol. 2018;93(2):205-211. doi:10.1590/ abd1806-4841.20186168

8. Salunke AS, Nagargoje MV, Belgaumkar VA, Tolat SN, Chavan RB. Association of metabolic syndrome in chronic plaque psoriasis patients and their correlation with disease severity, duration and age: a case control study from Western Maharashtra. J Clin Diagn Res. 2017;11(8):WC06-WC10. doi:10.7860/JCDR/2017/24390.10348

9. Singh S, Dogra S, Shafiq N, Bhansali A, Malhotra S. Prevalence of metabolic syndrome in psoriasis and levels of interleukin- 6 and tumor necrosis factor-alpha in psoriasis patients with metabolic syndrome: indian tertiary care hospital study. Int J Appl Basic Med Res. 2017;7(3):169-175. doi:10.4103/ijabmr.IJABMR_330_16

10. Uczniak S, Gerlicz ZA, Kozlowska M, Kaszuba A. Presence of selected metabolic syndrome components in patients with psoriasis vulgaris. Postepy Dermatol Alergol. 2016;33(2):114-119. doi:10.5114/ada.2016.59153

11. Vachatova S, Andrys C, Krejsek J, et al. Metabolic syndrome and selective inflammatory markers in psoriatic patients. J Immunol Res. 2016;2016:5380792. doi:10.1155/2016/5380792
12. Goolam Mahyoodeen N, Crowther NJ, Snyman T, Pillay L, Tikly M. High burden of the metabolic syndrome and its component disorders in South Africans with psoriasis. Int $J$ Dermatol. 2019;58 (5):557-562. doi:10.1111/ijd.14348

13. Farshchian M, Ansar A, Sobhan M. Associations between cardiovascular risk factors and psoriasis in Iran. Clin Cosmet Investig Dermatol. 2015;8:437-442. doi:10.2147/CCID.S86418

14. Gisondi P, Fostini AC, Fossa I, et al. Psoriasis and the metabolic syndrome. Clin Dermatol. 2018;36(1):21-28. doi:10.1016/j. clindermatol.2017.09.005

15. Radtke MA, Mrowietz U, Feuerhahn J, et al. Early detection of comorbidity in psoriasis: recommendations of the National Conference on Healthcare in Psoriasis. JDDG. 2015;13(7):674-690. doi: $10.1111 /$ ddg. 12643

16. Albareda M, Ravella A, Castello M, Saborit S, Peramiquel L, Vila L. Metabolic syndrome and its components in patients with psoriasis. Springerplus. 2014;3:612. doi:10.1186/2193-1801-3-612

17. Misra A, Wasir JS, Pandey RM. An evaluation of candidate definitions of the metabolic syndrome in adult Asian Indians. Diabetes Care. 2005;28(2):398-403. doi:10.2337/diacare.28.2.398

18. Fernandez-Armenteros JM, Gomez-Arbones X, Buti-Soler M, et al. Psoriasis, metabolic syndrome and cardiovascular risk factors. A population-based study. J Eur Acad Dermatol Venereol. 2019;33 (1):128-135. doi:10.1111/jdv.15159

19. Kim GW, Park HJ, Kim HS, et al. Analysis of cardiovascular risk factors and metabolic syndrome in korean patients with psoriasis. Ann Dermatol. 2012;24(1):11-15. doi:10.5021/ad.2012.24.1.11

20. Orgaz-Molina J, Magro-Checa C, Arrabal-Polo MA, et al. Association of 25-hydroxyvitamin D with metabolic syndrome in patients with psoriasis: a case-control study. Acta Derm Venereol. 2014;94(2):142-145. doi:10.2340/00015555-1642

21. Koku Aksu AE, Saracoglu ZN, Metintas S, Sabuncu I, Cetin Y. Age and gender differences in Framingham risk score and metabolic syndrome in psoriasis patients: a cross-sectional study in the Turkish population. Anatol $J$ Cardiol. 2017;17(1):66-72. doi:10.14744/AnatolJCardiol.2016.6679

22. Itani S, Arabi A, Harb D, et al. High prevalence of metabolic syndrome in patients with psoriasis in Lebanon: a prospective study. Int J Dermatol. 2016;55(4):390-395. doi:10.1111/ijd.12811

23. Kim HN, Han K, Park YG, Lee JH. Metabolic syndrome is associated with an increased risk of psoriasis: a nationwide population-based study. Metabolism. 2019;99:19-24. doi:10.1016/j.metabol.2019.07.001

24. Akcali C, Buyukcelik B, Kirtak N, et al. Clinical and laboratory parameters associated with metabolic syndrome in Turkish patients with psoriasis. J Int Med Res. 2014;42(2):386-394. doi:10.1177/0300060513502891

25. Espinoza Hernandez CJ, Lacy Niebla RM, Soto Lopez ME, et al. [Prevalence of metabolic syndrome in patients with psoriasis]. Gac Med Mex. 2014;150(4):311-316. Spanish.

26. Kokpol C, Aekplakorn W, Rajatanavin N, et al. Prevalence and characteristics of metabolic syndrome in South-East Asian psoriatic patients: a case-control study. J Dermatol. 2014;41(10):898-902. doi:10.1111/1346-8138.12614

27. Meziane M, Kelati A, Najdi A, et al. Metabolic syndrome in Moroccan patients with psoriasis. Int $J$ Dermatol. 2016;55 (4):396-400. doi:10.1111/ijd.12623

28. Kim CR, Lee JH. An observational study on the obesity and metabolic status of psoriasis patients. Ann Dermatol. 2013;25(4):440-444. doi:10.5021/ad.2013.25.4.440

29. Ghiasi M, Nouri M, Abbasi A, Hatami P, Abbasi MA, Nourijelyani K. Psoriasis and increased prevalence of hypertension and diabetes mellitus. Indian J Dermatol. 2011;56(5):533-536. doi:10.4103/0019-5154.87149

30. Langan SM, Seminara NM, Shin DB, et al. Prevalence of metabolic syndrome in patients with psoriasis: a population-based study in the United Kingdom. J Invest Dermatol. 2012;132(3 Pt 1):556-562. doi:10.1038/jid.2011.365 
31. Milcic D, Jankovic S, Vesic S, et al. Prevalence of metabolic syndrome in patients with psoriasis: a hospital-based cross-sectional study. An Bras Dermatol. 2017;92(1):46-51. doi:10.1590/abd18064841.20175178

32. Chan WMM, Yew YW, Theng TSC, Liew CF, Oon HH. Prevalence of metabolic syndrome in patients with psoriasis: a cross-sectional study in Singapore. Singapore Med J. 2019;61:194-199. doi:10.11622/smedj.2019152
33. Sondermann W, Djeudeu Deudjui DA, Korber A, et al. Psoriasis, cardiovascular risk factors and metabolic disorders: sex-specific findings of a population-based study. J Eur Acad Dermatol Venereol. 2019;34:779-786. doi:10.1111/jdv.16029

Diabetes, Metabolic Syndrome and Obesity: Targets and Therapy

\section{Publish your work in this journal}

Diabetes, Metabolic Syndrome and Obesity: Targets and Therapy is an international, peer-reviewed open-access journal committed to the rapid publication of the latest laboratory and clinical findings in the fields of diabetes, metabolic syndrome and obesity research. Original research, review, case reports, hypothesis formation, expert opinion and commentaries are all considered for publication. The manuscript management system is completely online and includes a very quick and fair peer-review system, which is all easy to use. Visit http://www.dovepress.com/testimonials.php to read real quotes from published authors. 